\title{
Phytotherapeutics oridonin and ponicidin show additive effects combined with irradiation in pancreatic cancer in vitro
}

\author{
Jakob Liermann 1 , Patrick Naumann¹, Franco Fortunato², Thomas E. Schmid ${ }^{3}$, \\ Klaus-Josef Weber ${ }^{1}$, Jürgen Debus ${ }^{3}$, Stephanie E. Combs ${ }^{3,4}$ \\ ${ }^{1}$ Department of Radiation Oncology, Heidelberg University Hospital, Heidelberg, Germany \\ ${ }^{2}$ Heidelberg University Hospital, Section Surgical Research, Heidelberg, Germany \\ ${ }^{3}$ Department of Radiation Oncology, Klinikum rechts der Isar, Technische Universität München, Munich, Germany \\ ${ }^{4}$ Institute of Innovative Radiotherapy (iRT), Department of Radiation Sciences (DRS), Helmholtz Zentrum München, \\ Neuherberg, Germany
}

Radiol Oncol 2017; 51(4): 407-414.

Received 28 June 2017

Accepted 13 October 2017

Correspondence to: Dr. Jakob Liermann, Department of Radiation Oncology, Heidelberg University Hospital, INF 400, 69120 Heidelberg, Germany. E-mail: jakob.liermann@med.uni-heidelberg.de

Disclosure: No potential conflicts of interest were disclosed.

*J Liermann and P Naumann contributed equally to this work.

Background. Chemoradiation of locally advanced non-metastatic pancreatic cancer can lead to secondary operability by fumor mass reduction. Here, we analyzed radiomodulating effects of oridonin and ponicidin in pancreatic cancer in vitro. Both agents are ent-kaurane diterpenoids, extracted from Isodon rubescens, a plant that is well known in Traditional Chinese Medicine. Cytotoxic effects have recently been shown in different tumor entities for both agents. Materials and methods. Pancreatic cancer cell lines AsPC-1, BXPC-3, Panc-1 and MIA PaCa-2 were pretreated with oridonin or ponicidin and irradiated with $2 \mathrm{~Gy}$ to $6 \mathrm{~Gy}$. Long-term survival was determined by clonogenic assay. Cell cycle effects and intensity of $\mathrm{YH} 2 \mathrm{AX}$ as indicator for DNA double-strand breaks were investigated by flow cytometry. Western blotting was used to study the DNA double-strand break repair proteins KU70, KU80 and XRCC4.

Results. Oridonin and ponicidin lead to a dose-dependent reduction of clonogenic survival and an increase in YH2AX. Combined with irradiation we observed additive effects and a prolonged G2/M-arrest. No relevant changes in the levels of the DNA double-strand break repair proteins were detected.

Conclusions. Pretreatment with oridonin or ponicidin followed by irradiation lead to an additional reduction in survival of pancreatic cancer cells in vitro, presumably explained by an induced prolonged G2/M-arrest. Both agents seem to induce DNA double-strand breaks but do not interact with the non-homologous end joining (NHEJ) pathway.

Key words: oridonin; ponicidin; irradiation; pancreatic cancer; $\mathrm{YH} 2 \mathrm{AX}$; radiosensitivity

\section{Introduction}

Pancreatic cancer is the fourth leading cause of cancer related deaths in the United States. ${ }^{1}$ Surgical resection is still the most important treatment modality and prognostic factor, but in the late stage of the disease, only $10-20 \%$ of all primary tumours are still operable. ${ }^{2}$ Beside primary metastasized and thus palliative stage IV disease $30-40 \%$ of all cases are locally advanced but non-metastasized tumors that could benefit from neoadjuvant treatment. ${ }^{3}$ We recently reported that approximately one third of these locally advanced tumors become resectable by a neoadjuvant combined chemoradiation and thus regain a curative therapy option. ${ }^{4}$ To further increase the efficacy of radiotherapy in pancreatic cancer, new chemotherapeutics, that can sensitize pancreatic cancer tissue to radiation, are needed. 
The diterpenoids oridonin and ponicidin are both extracts from Isodon rubescens, a plant used in Traditional Chinese Medicine that is known for its anti-inflammatory and antitumor properties in the treatment of esophageal and cardiac cancer. ${ }^{5}$ Recently, antineoplastic properties of oridonin and ponicidin have been published..$^{6-8}$ In pancreatic cancer oridonin induces apoptosis and leads to an arrest in the G2/M-phase of the cell cycle. ${ }^{9-12}$ Further, oridonin inhibits nuclear factor-kB. ${ }^{13}$ Ponicidin is also known to induce apoptosis ${ }^{14-17}$ but to our knowledge, there is no data for ponicidin in pancreatic cancer.

Potential radiosensitization could be observed in chinese-hamster-V79 cells by oridonin. ${ }^{18}$ Apart from this study we found no publication concerning oridonin or ponicidin induced radiomodulation.

As natural occurring products for both substances no serious side effects in humans are known which qualifies them as potential new chemotherapeutic drugs. Data of an in vivo trial for oridonin used in acute leukemia seems to confirm this hypothesis. ${ }^{19}$ Here, we analyzed radiomodulating effects of oridonin and ponicidin in pancreatic cancer cells in vitro by clonogenic assay, cell cycle analysis, $\gamma \mathrm{H} 2 \mathrm{AX}$ expression and DNA repair proteins expression analysis.

\section{Material and methods}

\section{Cell culture and irradiation}

The pancreatic cancer cell lines AsPC-1, BxPC-3 and MIA PaCa-2 were cultured in RPMI-1640 medium, the cell line Panc-1 in Dulbecco's modified Eagle's Medium (DMEM). Media included 10\% FBS and 1\% Penicillin/Streptomycin. The cells were cultured at $37^{\circ} \mathrm{C}$ in $5 \% \mathrm{CO}_{2}$. AsPC-1 and Panc-1 cell lines were purchased from CLS cell line service $\mathrm{GmbH}$, Germany and BxPC-3 as well as MIA PaCa-2 cells from LGC Standards GmbH (ATCC), Germany. Oridonin was obtained from SigmaAldrich Corporation, USA and ponicidin from Shanghai Zhanshu Chemical Technology Co., Ltd., China. Both reagents were dissolved in DMSO and stored at $-80^{\circ} \mathrm{C}$.

Cells were X-irradiated (X-RAD 320, Precision X-Ray, Inc., USA) operated at $320 \mathrm{kV}$ and $12.5 \mathrm{~mA}$. The average dose rate was $1.1 \mathrm{~Gy} / \mathrm{min}$. The used filter consisted of $1.5 \mathrm{~mm} \mathrm{Al}, 0.25 \mathrm{~mm} \mathrm{Cu}$ and 0.75 $\mathrm{mm} \mathrm{Sn}$. The tissue culture flasks, in which the cells were cultured, were placed on a PMMA-plate of 1 $\mathrm{cm}$ height.

\section{Clonogenic assay}

To assess long-term cell survival, a defined number of cells were seeded in $25 \mathrm{~cm}^{2}$ tissue culture flasks and incubated for 24 hours. Cell counts were adjusted for the different treatment schemes. Cell lines AsPC-1, BxPC-3 and Panc-1 were treated with oridonin, ponicidin or DMSO (control) for 24 hours as indicated and irradiated afterwards once with $2 \mathrm{~Gy}$ or 6 Gy. The dose of each substance and the intensity of irradiation were established through pre-experiments to achieve a survival rate of $20-70 \%$ after single treatment alone. MIA PaCa-2 cells were pretreated with lower doses of oridonin and ponicidin due to higher sensitivity to the reagents. 8-9 days (time differs among cell lines) after the treatment cells were fixed in $70 \%$ ethanol and stained with $0.2 \%$ methylenblue. Colonies, defined as containing at least 50 cells, were counted manually under a light microscope and the plating efficiency, defined as the quotient of counted colonies to plated cells, was determined. The surviving fraction was defined as the ratio of each experiment's plating efficiency to its control. Cells were plated in triplets to compensate for pipetting errors and at least three independent experiments were performed.

\section{Determination of radiosensitivity}

To analyze combined treatments, surviving fractions were normalized to the values of corresponding single reagent treatment using a ratio of the combined treatment's plating efficiency to the single reagent treatment one's. Dose-response curves were created using the linear-quadratic model..$^{20}$

Combination effects were analyzed defining additivity according to Steel and Peckham. ${ }^{21}$ For this purpose a dose-response curve was calculated that represents the course of the measured control curve at a pre-effect similar to the single reagent treatment's effect ("theoretical control-curve“). Additive effects were defined as area between the measured control curve and the theoretical one. Supraadditive effects were defined as beneath the theoretical control curve and radioprotective effects as above the measured control curve. Independent toxicity was defined as identical curve to the measured control curve (for further information see Suppl. Figure 1).

\section{Cell cycle analysis}

Since impact on cell cycle distribution was regressing 24 hours after pretreatment a shorter exposi- 
(A)
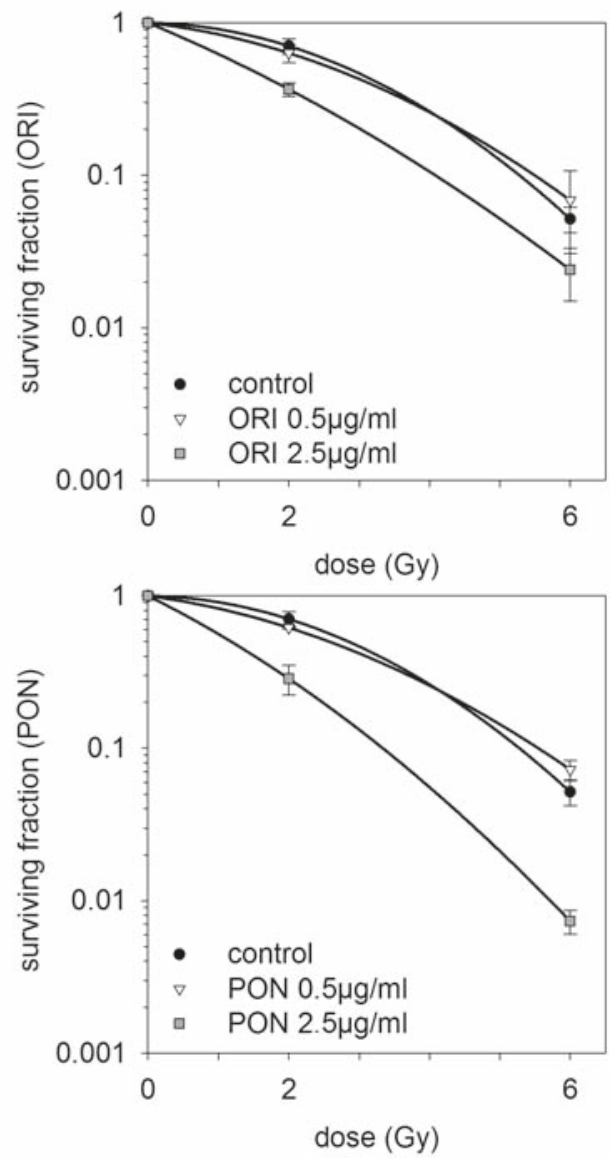

(B)
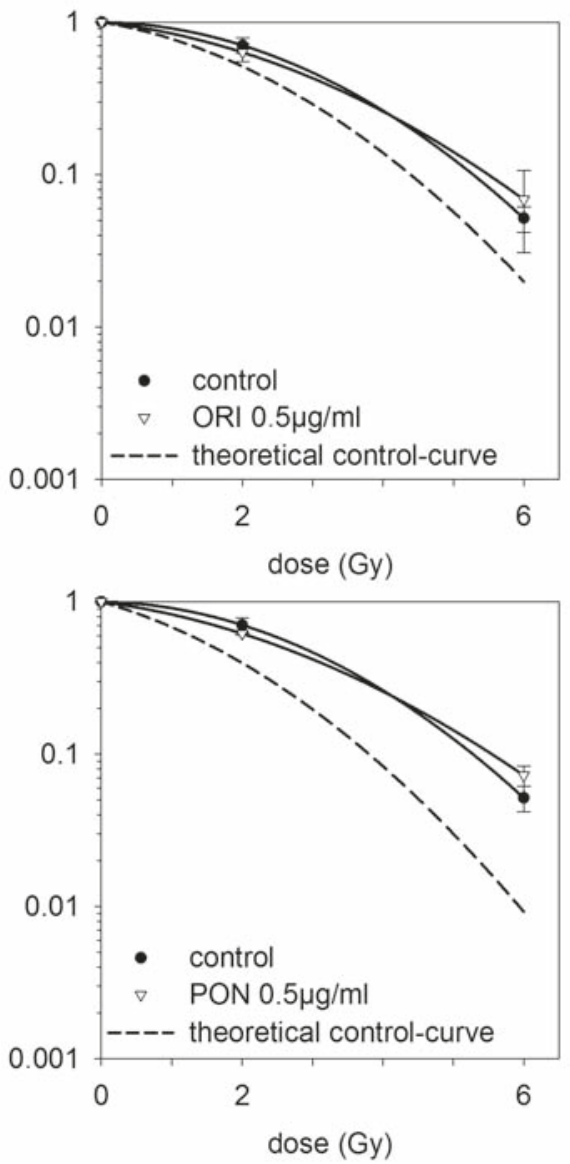

Panc-1
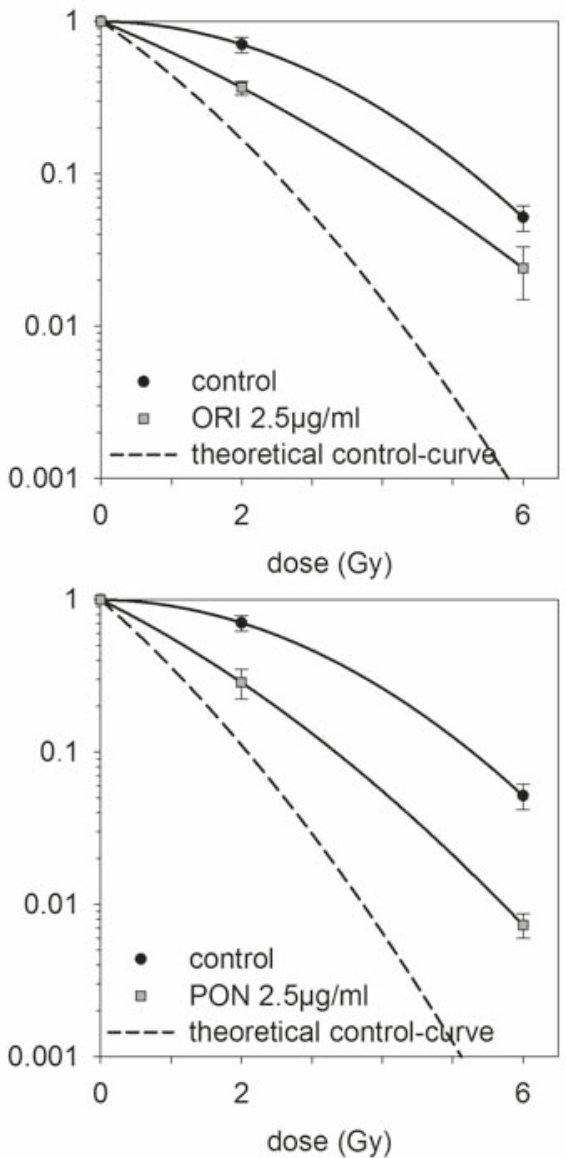

FIGURE 1. Clonogenic survival of Panc-1 cells treated with either irradiation and/or oridonin (ORI) and ponicidin (PON) as indicated. (A) survival curves and $(B)$ radiosensitivity to different dosages with measured and theoretical control curve, according to Steel and Peckham for Panc-1 cells. Results are presented as mean values +/- standard deviation. At least three independent experiments were performed.

tion time to oridonin and ponicidin compared to the other experiments was chosen. Simultaneously the dosage was augmented to compensate for shortage in exposition time. Cell lines Panc-1 and MIA PaCa-2 were pretreated for two hours with oridonin, ponicidin or DMSO (control) and irradiated as indicated. To induce a less lethal damage than one would expect of $6 \mathrm{~Gy}$, an irradiation dose of 4 Gy was chosen. Cells were then fixed in ice-cold ethanol and RNA-strands were cleaved by RNase A (AppliChem GmbH, Germany) at the following time points: beginning of the treatment, before irradiation, 12 and 24 hours after irradiation. Then cells were stained with propidium iodide (Sigma-Aldrich Corporation, USA) and the amount of propidium iodide per cell was quantified in a flow cytometer (FACScan, Becton Dickinson and Company, USA). At least $10^{4}$ ungated cells were analyzed by BD CellQuest Pro 4.0.2 (Becton
Dickinson and Company, USA) and the different phases of the cell cycle were determined using the software ModFit LT 3.0 (Verity Software House, Inc., USA). At least two independent experiments were performed.

\section{YH2AX-quantification}

Since clonogenic assays revealed different sensitivity between AsPC-1, BxPC-3 and Panc- 1 cells on the one hand and MIA PaCa-2 cells on the other hand further experiments were done with the cell lines Panc-1 and MIA PaCa-2. To quantify the induction of DNA double-strand breaks Panc- 1 and MIA PaCa-2 cells were pretreated with oridonin, ponicidin or DMSO (control) for 24 hours and were irradiated once as indicated. At different time points after irradiation MIA PaCa-2 and Panc-1 cells were fixed using 1\% PFA and ice-cold ethanol. Cells 
were then stained with a $\gamma \mathrm{H} 2 \mathrm{AX}$-antibody (art. no. 16-202A, Merck KGaA, Darmstadt, Germany) and pan-nuclear intensity was measured using a flow cytometer. Results were normalized by creating a ratio of the measured intensity to the intensity of the control probes. At least three independent experiments were performed.

\section{Western Blot}

Cell lines Panc-1 and MIA PaCa-2 were treated with oridonin, ponicidin or DMSO (control) for 24 hours. Then lysates of the cells were gained using lysis buffer (10 mM HEPES pH 7.4, 5 mM CHAPS, $5 \mathrm{mM}$ DTT) and inhibitors (PMSF, Pepstatin, Leupeptin, Aprotinin). The protein concentration was determined in a Bradford protein assay and 20 $\mu \mathrm{g}$ to $30 \mu \mathrm{g}$ Protein were used for Western Blots. A SDS-polyacrylamide-gel-electrophoresis was run using $12 \%$ polyacrylamide-gels with a voltage of $160 \mathrm{~V}$ and amperage of $250 \mathrm{~mA}$. A wet transfer was performed $(80 \mathrm{~V}, 60 \mathrm{~mA})$ to transfer the proteins on a polyvinylidenfluoride membrane. The membrane was blocked using Roti-Block (Carl Roth. GmbH \& Co. KG, Germany). b-Actin (art. no. 4967), GAPDH (art. no. 2118), Ku70 (art. no. 4588) and Ku80 (art. no 2753) primary antibodies were purchased from Cell signaling Technology Inc., USA. XRCC4 (art. no. sc-271087) and secondary antibodies (art. no. sc-2380, sc-2379) were obtained from Santa Cruz Biotechnology, Inc., USA. Dilution was done according to manufacturer's instructions. X-Ray films were scanned and the density of the protein bands were determined with the software Image J 1.47V (National Institute of Health, USA). Density was normalized by creating a ratio of protein band to house-keeping protein's one to avoid minimal differences in the amount of protein load. Then another ratio of the treatment's value to its control was calculated and allowed to quantify densities and treatment differences. At least two independent experiments were performed.

\section{Statistical analysis}

The results of each experiment were generated as described above. Statistical analysis of the results was done with an unpaired two-tailed t-test using Microsoft ${ }^{\circledR}$ Excel® 2008 for Mac 12.3.6 (Microsoft Corporation, USA). The dose control curves and the figures were calculated and generated with SigmaPlot 12.0 (Systat Software Inc., USA). Results are presented as mean values +/- standard deviation. $\mathrm{P}<0.05$ was considered as a significant differ- ence between the compared results. ${ }^{*} \mathrm{P}<0.05,{ }^{* *} \mathrm{P}<$ $0.005,{ }^{* * *} \mathrm{P}<0.001$.

\section{Results}

Oridonin and ponicidin reduce clonogenic survival and show additive effects when combined with irradiation

To investigate long-term survival cells were treated with oridonin or ponicidin in a low dosage of 0.5 $\mu \mathrm{g} / \mathrm{ml}$ and high dosage of $2.5 \mu \mathrm{g} / \mathrm{ml}$ (Figure 1A). Since MIA PaCa-2 cells exposed a higher sensitivity to both oridonin and ponicidin, we used $0.1 \mu \mathrm{g} /$ $\mathrm{ml}$ and $0.5 \mu \mathrm{g} / \mathrm{ml}$ as the low and the high dosage in these cells, respectively. MIA PaCa-2 cells showed a significant reduction in clonogenic survival to a fraction of $51 \%$ when treated with the lower dosage of oridonin and thus were much more sensitive than the other cell lines, with a mean surviving fraction of $88 \%$ in AsPC-1, BxPC-3 and Panc-1 cells (Suppl. Figure 2). Interestingly, ponicidin was more effective than oridonin: $2.5 \mu \mathrm{g} / \mathrm{ml}$ of ponicidin lead to a mean surviving fraction of $12 \%$. The same amount of oridonin showed an almost threefold higher surviving fraction of 32\% in AsPC-1-, BxPC-3- and Panc-1 cells (Suppl. Figure 3).

A further dose-dependent reduction in survival could be observed by combination with irradiation. The mean surviving fraction of $88 \%$ after single treatment with low dosage of oridonin could be further attenuated by additional irradiation with 2 Gy or 6 Gy to mean $63 \%$ and $13 \%$, respectively.

Using these results we investigated the radiosensitivity according to the model of Steel and Peckham as described in the methods section. ${ }^{21}$ The higher dose of oridonin and ponicidin lead to additive effects in almost all investigated cell lines when combined with 2 Gy or 6 Gy irradiation, whereas the lower dose just showed independent toxicity (Figure 1B and Suppl. Figure 4). Radioprotectivity as well as supraadditivity could not be observed.

\section{G2/M-arrest after combined treatment of radiation and oridonin or ponicidin}

To determine changes in cell cycle, the four pancreatic cancer cell lines were treated with oridonin or ponicidin alone as well as in combination with irradiation. Cell cycle phases were analyzed 12 and 24 hours after irradiation. Pretreatment with oridonin (Figure 2A) or ponicidin (Figure 2B) induced a clear G2/M-arrest of the cell lines AsPC-1 and Panc-1 after 12 hours with up to $36 \%$ (Figure 2 and Suppl. 
Figure 5). The G2/M- fraction of combined treatments was not as high as the one observed after irradiation alone but was augmented in time leading to the fact that 24 hours after irradiation there still was a clear G2/M-arrest in the cell lines AsPC-1, BcPC-3 and Panc- 1 when treated with the combined therapy. MIA PaCa-2 cells showed a clear G2/M-arrest 24 hours after combined treatment with ponicidin but not with oridonin (Suppl. Figure 5). We did not observe a sub-G1 fraction as surrogate of apoptosis.

\section{Oridonin and poncidin induce an increased pan-nuclear $\mathrm{YH} 2 \mathrm{AX}$-intensity}

The potential influence of oridonin or ponicidin on the DNA double-strand break repair was analyzed by measuring the pan-nuclear $\gamma \mathrm{H} 2 \mathrm{AX}$-intensity of pretreated cells via flow cytometry. Both oridonin (Figure $3 \mathrm{~A}$ ) and poncidin (Figure $3 \mathrm{~B}$ ) lead to an approximately 2.5-fold increase of the $\gamma \mathrm{H} 2 \mathrm{AX}$ intensity shortly after treatment, independent of the particular dose level $(7.5 \mu \mathrm{g} / \mathrm{mL}$ vs. $15 \mu \mathrm{g} / \mathrm{mL})$. There was an increase of $\gamma \mathrm{H} 2 \mathrm{AX}$ after irradiation alone but highest $\gamma \mathrm{H} 2 \mathrm{AX}$-intensities could be observed in combined treatments. The Intensity of $\gamma \mathrm{H} 2 \mathrm{AX}$ already declined 10 hours posttreatment. We could not observe a difference in the relative reduction of the $\gamma \mathrm{H} 2 \mathrm{AX}$-intensity between irradiation alone and combined treatments.

\section{Oridonin and ponicidin do not influence the expression of DNA repair proteins related to the non-homologous end joining (NHEJ) pathway}

To determine the influence of oridoinin or ponicidin on the repair of DNA double-strand breaks, Panc-1- and MIA PaCa-2-cells were treated with either agent and three main proteins of the NHEJ pathway, namely XRCC4, Ku70 and Ku80 were detected by Western Blots. Pretreatment with oridonin or ponicidin lead to a slight reduction of XRCC4-expression but there was no considerable change in Ku70 and Ku80 protein expression in MIA PaCa-2 cells. Panc-1 cells showed no stringent effect in XRCC4 protein expression but a dose-dependent increase of Ku70 and Ku80 after pretreatment with oridonin or ponicidin (Figure 4).

\section{Discussion}

The aim of this study was to investigate the two phytotherapeutics oridonin and ponicidin for their
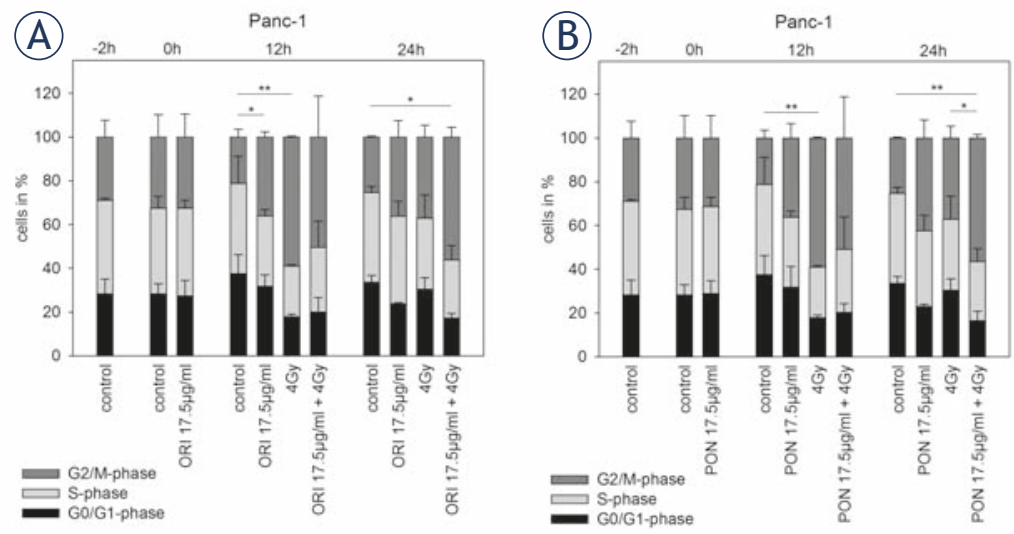

FIGURE 2. Cell cycle assay of Panc-1 cells treated with irradiation and/or (A) oridonin (ORI) and (B) ponicidin (PON) as indicated. Results are presented as mean values +/- standard deviation. ${ }^{*} \mathrm{P}<0.05,{ }^{*} \mathrm{P}<0.005$, asterisk referring to the amount of cells in G2/M-phase. At least two independent experiments were performed.
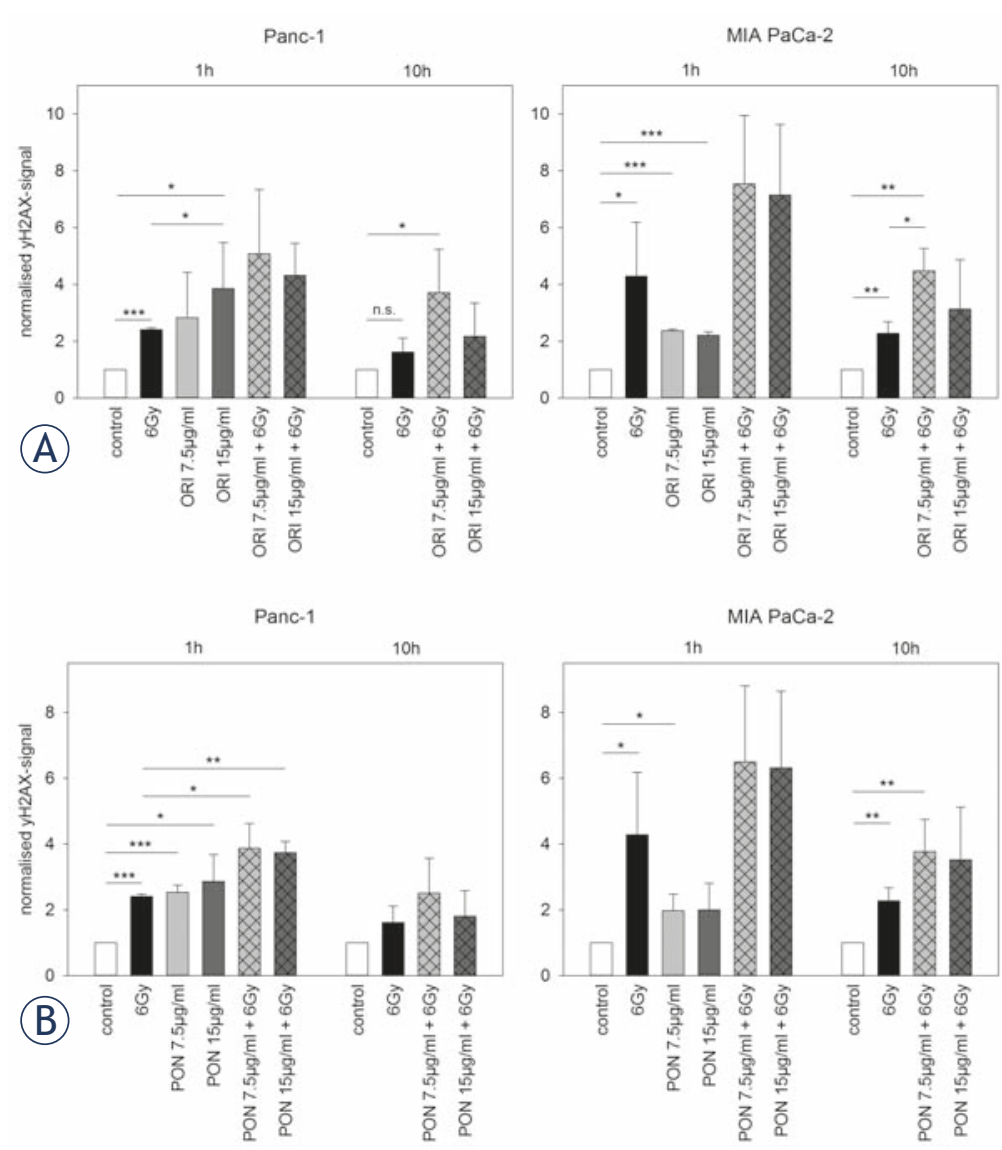

FIGURE 3. YH2AX-intensity of Panc-1 and MIA PaCa-2 cells treated with irradiation and/or (A) oridonin (ORI) and (B) ponicidin (PON) as indicated. Results are presented as mean values $+/$ - standard deviation. ${ }^{*} P<0.05,{ }^{* *} \mathrm{P}<0.005,{ }^{* * * P}<0.001$, n.s. $=$ not significant. At least three independent experiments were performed. 

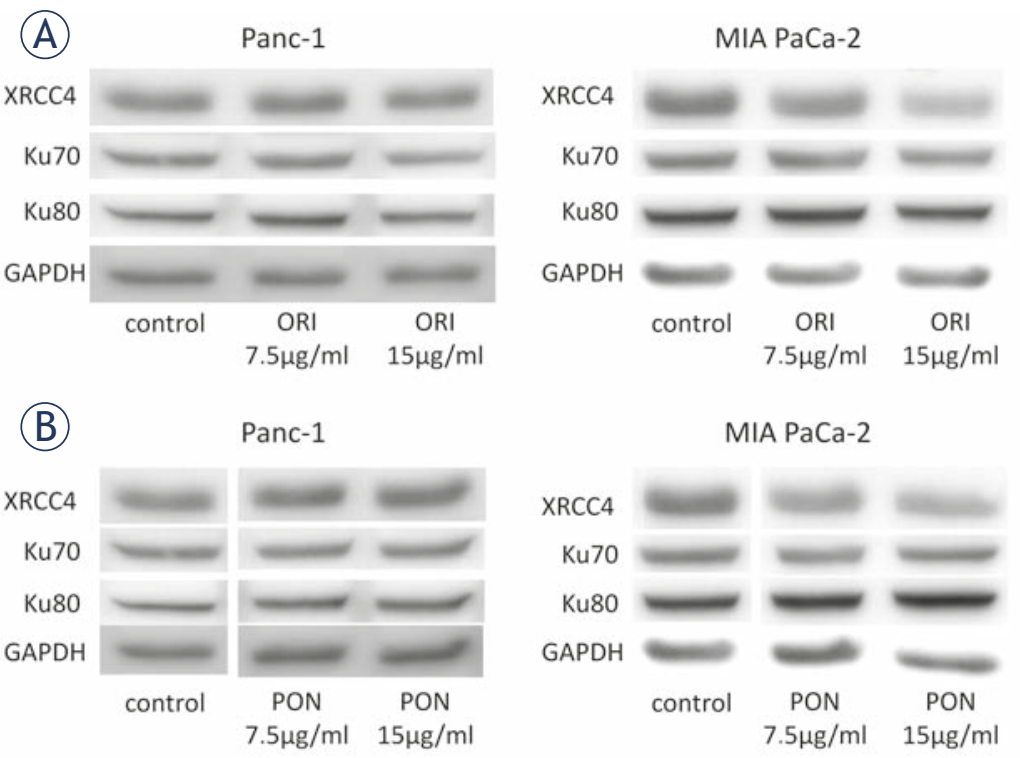

FIGURE 4. Western Blots of Panc-1 and MIA PaCa-2 cells treated with either (A) oridonin (ORI) and (B) ponicidin (PON) as indicated. One representative Western Blot is shown. At least two independent experiments were performed. NHEJ = nonhomologous end-joining.

capability to enhance effects of irradiation in pancreatic cancer cells. Compared to other tumor entities pancreatic cancer cells are more resistant to chemo- and radiotherapy and thus radiosensitizing agents are highly clinically relevant.

To investigate treatment efficacy long-term survival was determined by clonogenic assays. Changes in cell cycle distribution and intensity of $\gamma \mathrm{H} 2 \mathrm{AX}$ as indicator for DNA double-strand breaks were investigated by flow cytometry, since effects of radiotherapy base predominantly on induction of DNA double-strand breaks. ${ }^{22}$ Western blotting was used to study key proteins of the NHEJ pathway of the DNA double-strand break repair. To our knowledge we are the first who tested ponicidin in combination with irradiation whereas potential radiosensitzing effects of oridonin could already be observed in chinese-hamster-V79 cells. ${ }^{18}$

After treatment with either oridonin or ponicidin we observed a dose-dependent reduction of clonogenic survival in all tested pancreatic cancer cell lines AsPC-1, BxPC-3, MIA PaCa-2 and Panc-1 after treatment with either oridonin or ponicidin. Compared to oridonin, equal doses of ponicidin were more effective in reducing clonogenic survival. Similar results were observed by Hsieh et al. in clonogenic survival assays of the breast cancer cell line MCF-7, who also tested both agents in proliferation assays with comparable results of oridonin and ponicidin. ${ }^{23}$ Based on these data we infer that ponicidin might be a more potent cytostatic agent than oridonin.

After combined treatment with irradiation and different concentrations of oridonin or ponicidin we observed an increased reduction of clonogenic survival. The analysis of potential interactions revealed a trend towards additive effects for the higher concentration of oridonin as well as ponicidin. Supraadditive effects that provide evidence for actual radiosensitization could not be observed.

Cell cycle assays of the present study showed that both oridonin and ponicidin lead to an accumulation of cells in the G2/M-phase of the cell cycle. The combination of irradiation and oridonin or ponicidin leads to an even prolonged G2/M-arrest. Cells that are stuck in this phase usually present DNA-lesions, such as DNA double-strand breaks or blocked DNA replication forks, which have to be repaired before passing the G2/M-checkpoint. ${ }^{24}$ An oridonin-induced G2/M-arrest was previously reported by Qi et al. for the pancreatic cancer cell line Panc- 1 along with a suppression of cell cycle regulating cyclin $\mathrm{B} 1$ and cdc2. ${ }^{10}$ In our study, we focused on DNA double-strand breaks as underlying mechanism especially after combination with irradiation. Therefore we did not investigate any changes in proteins of the cell cycle regulation but further experiments should pinpoint the precise mode G2/M-phase arrest induced by oridonin and ponicidin. Since induction of apoptosis was reported for both oridonin and ponicidin ${ }^{9-12,14-17}$, we expected a sub-G1 peak in cell cycle analysis but could not observe any peak in the sub-G1 noise. Interestingly, the cell cycle assays of Qi et al. for PANC-1 cells treated with oridonin also did not show any sub-G1 peak. ${ }^{10}$

The analyzed pan-nuclear $\gamma \mathrm{H} 2 \mathrm{AX}$-quantification revealed a two-fold higher $\gamma \mathrm{H} 2 \mathrm{AX}$-intensity after treatment with oridonin or ponicidin. This increase in $\gamma \mathrm{H} 2 \mathrm{AX}$ level was independent of the particular dose level of oridonin and ponicidin. Combined with irradiation both oridonin and ponicidn lead to an even higher amount of $\gamma \mathrm{H} 2 \mathrm{AX}$. Pan-nuclear determination of $\gamma \mathrm{H} 2 \mathrm{AX}$ is just an indirect indicator of DNA double-strand break induction, but it is comparable to the quantification of $\gamma \mathrm{H} 2 \mathrm{AX}-$ foci $^{25}$ which is known to be highly suspect for an induction of DNA double-strand breaks. ${ }^{26}$ Accordingly, oridonin and ponicidin as well as irradiation seem to induce DNA double-strand breaks. Beside possible effects on cell cycle phase proteins as suggested by Qi et al..$^{10}$ the induction of DNA double-strand breaks are a good explanation for the observed G2/M-arrest. An already described augmentation 
of $\gamma \mathrm{H} 2 \mathrm{AX}$ in oridonin-treated BxPC-3 cells and an increased $\gamma \mathrm{H} 2 \mathrm{AX}$-concentration in breast cancer cells exposed to irradiation are in line with our present data..$^{9,27}$

By comparison of $\gamma \mathrm{H} 2 \mathrm{AX}$-intensities at different time points the level of $\gamma \mathrm{H} 2 \mathrm{AX}$ of the combined treatment schemes decreased quite similar to that of the irradiation treatment alone. We therefore have to conclude that oridonin and ponicidin do not significantly lower the DNA double-strand break repair capacity. To verify this, proteins of the DNA double-strand break repair were detected by Western Blot. Since pancreatic cancer cells are known to show only weak activity of the homologous recombination pathway ${ }^{28}$, we concentrated on the key proteins of the NHEJ. There were no relevant changes in the amount of Ku70, Ku80 or XRCC4 in oridonin- or ponicidin-treated cells supporting the hypothesis that oridonin and ponicidin do not reduce the DNA double-strand break repair mechanisms. Further studies should investigate if oridonin or ponicidin interfere with proteins of the homologous recombination pathway of DNA double-strand break repair, such as RAD51 or MRE11/ RAD50/NBS1 complex.

Recently, Wang et al. could show synergistic effects of an oridonin derivative and paclitaxel through increased reactive oxygen species (ROS) levels and subsequent apoptosis induction..$^{29} \mathrm{Cao}$ et al. observed a synergistic effect of cetuximab and oridonin through increased ROS levels and epidermal growth factor receptor (EGFR) inhibition. ${ }^{30}$ Activation of ROS is known to be one of the main effects of irradiation in cancer treatment. These findings strengthen the idea of combined treatment schemes with oridonin and irradiation.

In the next step, both substances should be tested in combination with the recent standard chemotherapies such as gemcitabine or FOLFIRINOX. Finally, in vivo data are needed to reveal if efficacy of irradiation in pancreatic cancer patients can be improved by oridonin and ponicidin.

\section{Conclusions}

The current data demonstrate new aspects of the two interesting phytotherapeutics oridonin and poncidin. In vitro both substances are highly effective against pancreatic cancer cells especially when combined with irradiation. Therefore both oridonin and ponicidin are promising agents in pancreatic cancer therapy. Our data warrant further in vivo investigations especially in combination with irradiation or standard chemotherapy such as FOLFIRINOX or gemcitabine and nab-paclitaxel.

\section{Acknowledgements}

We thank Lena Orschiedt, Sigrid Daffinger, Sylvia Trinh and Ludmilla Frick for the excellent technical assistance with the experiments.

The datasets used and/or analysed during the current study are available from the corresponding author on reasonable request.

JL and PN designed and performed the experiments. JL and PN wrote the manuscript. TES performed the $\gamma \mathrm{H} 2 \mathrm{AX}$-quantification. FF, SEC, KJW and JD helped finalizing the manuscript. All authors read and approved the final manuscript.

We acknowledge financial support by Deutsche Forschungsgemeinschaft and Ruprecht-KarlsUniversität Heidelberg within the funding programme Open Access Publishing.

\section{References}

1. Siegel RL, Miller KD, Jemal A. Cancer statistics, 2015. CA Cancer J Clin 2015; 65: 5-29. doi: 10.3322/caac.21254

2. Kleeff J, Friess $\mathrm{H}$, Buchler MW. Neoadjuvant therapy for pancreatic cancer Br J Surg 2007; 94: 261-2. doi: 10.1002/bjs.5737

3. Gillen S, Schuster T, Meyer Zum Buschenfelde C, Friess H, Kleeff J. Preoperative/neoadjuvant therapy in pancreatic cancer: a systematic review and meta-analysis of response and resection percentages. PLoS Med 2010; 7: e1000267. doi: 10.1371/journal.pmed.1000267

4. Naumann P, Habermehl D, Welzel T, Debus J, Combs SE. Outcome after neoadjuvant chemoradiation and correlation with nutritional status in patients with locally advanced pancreatic cancer. Strahlenther Onkol 2013; 189: 745-52. doi: 10.1007/s00066-013-0393-3

5. Han QB, Li ML, Li SH, Mou YK, Lin ZW, Sun HD. Ent-kaurane diterpenoids from Isodon rubescens var. lushanensis. Chem Pharm Bull (Tokyo) 2003; 51: 790-3.

6. Owona BA, Schluesener HJ. Molecular insight in the multifunctional effects of oridonin. Drugs R D 2015; 15: 233-44. doi: 10.1007/s40268-015-0102-z

7. Tian W, Chen SY. Recent advances in the molecular basis of anti-neoplastic mechanisms of oridonin. Chin J Integr Med 2013; 19: 315-20. doi: 10.1007/ s11655-013-1437-3

8. Bai N, He K, Zhou Z, Tsai ML, Zhang L, Quan Z, et al. Ent-kaurane diterpenoids from Rabdosia rubescens and their cytotoxic effects on human cancer cell lines. Planta Med 2010; 76: 140-5. doi: 10.1055/s-0029-1186002

9. Xu B, Shen W, Liu X, Zhang T, Ren J, Fan Y, et al. Oridonin inhibits BxPC-3 cell growth through cell apoptosis. Acta Biochim Biophys Sin (Shanghai) 2015; 47: 164-73. doi: 10.1093/abbs/gmu134

10. Qi X, Zhang D, Xu X, Feng F, Ren G, Chu Q, et al. Oridonin nanosuspension was more effective than free oridonin on $\mathrm{G} 2 / \mathrm{M}$ cell cycle arrest and apoptosis in the human pancreatic cancer PANC-1 cell line. Int J Nanomedicine 2012; 7: 1793-804. doi: 10.2147/IJN.S29483

11. Bu HQ, Liu DL, Wei WT, Chen L, Huang $H$, Li Y, et al. Oridonin induces apoptosis in SW1990 pancreatic cancer cells via p53- and caspase-dependent induction of p38 MAPK. Oncol Rep 2014; 31: 975-82. doi: 10.3892/ or.2013.2888 
12. Bu HQ, Luo J, Chen H, Zhang JH, Li HH, Guo HC, et al. Oridonin enhances antitumor activity of gemcitabine in pancreatic cancer through MAPK-p38 signaling pathway. Int J Oncol 2012; 41: 949-58. doi: 10.3892/ijo.2012.1519

13. Ikezoe T, Yang Y, Bandobashi K, Saito T, Takemoto S, Machida H, et al. Oridonin, a diterpenoid purified from Rabdosia rubescens, inhibits the proliferation of cells from lymphoid malignancies in association with blockade of the NF-kappa B signal pathways. Mol Cancer Ther 2005; 4: 578-86. doi: 10.1158/1535-7163.MCT-04-0277

14. Liu JJ, Huang RW, Lin DJ, Peng J, Zhang M, Pan X, et al. Ponicidin, an entkaurane diterpenoid derived from a constituent of the herbal supplement PC-SPES, Rabdosia rubescens, induces apoptosis by activation of caspase-3 and mitochondrial events in lung cancer cells in vitro. Cancer Invest 2006; 24: 136-48. doi: 10.1080/07357900500524371

15. Liu JJ, Zhang Y, Guang WB, Yang HZ, Lin DJ, Xiao RZ. Ponicidin inhibits monocytic leukemia cell growth by induction of apoptosis. Int J Mol Sci 2008; 9: 2265-77. doi: 10.3390/ijms9112265

16. Zhang JF, Liu PQ, Chen GH, Lu MQ, Cai CJ, Yang Y, et al. Ponicidin inhibits cell growth on hepatocellular carcinoma cells by induction of apoptosis. Dig Liver Dis 2007; 39: 160-6. doi: 10.1016/j.dld.2006.09.011

17. Liu YF, Lu YM, Qu GQ, Liu Y, Chen WX, Liao XH, et al. Ponicidin induces apoptosis via JAK2 and STAT3 signaling pathways in gastric carcinoma. Int J Mol Sci 2015; 16: 1576-89. doi: 10.3390/ijms16011576

18. Murayama C, Nagao Y, Sano S, Ochiai M, Fuji K, Fujita E, et al. Effect of oridonin, a Rabdosia diterpenoid, on radiosensitization with misonidazole. Experientia 1987; 43: 1221-3.

19. Zhou GB, Kang H, Wang L, Gao L, Liu P, Xie J, et al. Oridonin, a diterpenoid extracted from medicinal herbs, targets AML1-ETO fusion protein and shows potent antitumor activity with low adverse effects on $t(8 ; 21)$ leukemia in vitro and in vivo. Blood 2007; 109: 3441-50. doi: 10.1182/ blood-2006-06-032250

20. Denekamp J, Waites T, Fowler JF. Predicting realistic RBE values for clinically relevant radiotherapy schedules. Int J Radiat Biol 1997; 71: 681-94.

21. Steel GG, Peckham MJ. Exploitable mechanisms in combined radiotherapychemotherapy: the concept of additivity. Int J Radiat Oncol Biol Phys 1979; 5: 85-91.

22. Morgan MA, Lawrence TS. Molecular pathways: overcoming radiation resistance by targeting DNA damage response pathways. Clin Cancer Res 2015; 21: 2898-904. doi: 10.1158/1078-0432.CCR-13-3229

23. Hsieh TC, Wijeratne EK, Liang JY, Gunatilaka AL, Wu JM. Differential contro of growth, cell cycle progression, and expression of NF-kappaB in human breast cancer cells MCF-7, MCF-10A, and MDA-MB-231 by ponicidin and oridonin, diterpenoids from the chinese herb Rabdosia rubescens. Biochem Biophys Res Commun 2005; 337: 224-31. doi: 10.1016/j.bbrc.2005.09.040

24. Roos WP, Kaina B. DNA damage-induced cell death: from specific DNA lesions to the DNA damage response and apoptosis. Cancer Lett 2013; 332: 237-48. doi: 10.1016/j.canlet.2012.01.007

25. MacPhail SH, Banath JP, Yu TY, Chu EH, Lambur H, Olive PL. Expression of phosphorylated histone $\mathrm{H} 2 \mathrm{AX}$ in cultured cell lines following exposure to X-rays. Int J Radiat Biol 2003; 79: 351-8.

26. Sedelnikova OA, Rogakou EP, Panyutin IG, Bonner WM. Quantitative detection of (125)IdU-induced DNA double-strand breaks with gamma-H2AX antibody. Radiat Res 2002; 158: 486-92.

27. Zhang $T$, Tan $Y$, Zhao R, Liu Z. DNA damage induced by oridonin involves cell cycle arrest at G2/M phase in human MCF-7 cells. Contemp Oncol (Pozn) 2013; 17: 38-44. doi: 10.5114/wo.2013.33772

28. Li YH, Wang X, Pan Y, Lee DH, Chowdhury D, Kimmelman AC. Inhibition of non-homologous end joining repair impairs pancreatic cancer growth and enhances radiation response. PLoS One 2012; 7: e39588. doi: 10.1371/ journal.pone.003958

29. Wang SQ, Wang C, Chang LM, Zhou KR, Wang JW, Ke Y, et al. Geridonin and paclitaxel act synergistically to inhibit the proliferation of gastric cancer cells through ROS-mediated regulation of the PTEN/PI3K/Akt pathway. Oncotarget 2016; 7: 72990-3002. doi: 10.18632/oncotarget.12166

30. Cao S, Xia M, Mao Y, Zhang Q, Donkor PO, Qiu F, et al. Combined oridonin with cetuximab treatment shows synergistic anticancer effects on laryngeal squamous cell carcinoma: involvement of inhibition of EGFR and activation of reactive oxygen species-mediated JNK pathway. Int J Oncol 2016; 49: 2075-87. doi: 10.3892/ijo.2016.3696 